\section{ANTRACOLOGÍA Y MÉTODOS: IMPLICACIONES EN LA ECONOMÍA PREHISTÓRICA, ETNOARQUEOLOGÍA Y PALEOECOLOGÍA}

\author{
CHARCOAL ANALYSIS AND METHODS: \\ IMPLICATIONS IN PREHISTORIC \\ ECONOMY, ETHNOARCHEOLOGY \\ AND PALEOECOLOGY
}

\section{PALOMA UZQUIANO (*)}

\section{RESUMEN}

Se define la Antracología aplicada a la Arqueología exponiendo detalladamente todas las etapas que constituyen su metodología de estudio. Desde la recogida de carbones en el yacimiento hasta la interpretación de los resultados en el laboratorio, esta disciplina ha revelado las múltiples implicaciones en áreas diversas de las Ciencias de la Tierra (Paleoecología) y de las Ciencias Históricas (Economía y Etnología prehistóricas).

\begin{abstract}
This paper concerns Anthracology (Charcoal analysis) from archaeological deposits. The methodology of study is discussed in detail. From sampling of charcoals in the site until their interpretation at the laboratory, this discipline has many implications in different areas both of the Earth Sciences and the Historical Sciences (Palaeoecology, Palaeoeconomy, Palaeoethnology).
\end{abstract}

Palabras clave: Antracología. Metodología. Etnoarqueología. Economía Prehistórica. Paleoecología.

Key words: Charcoal analysis. Methodology. Ethnoarchaeology. Prehistoric Economy. Palaeoecology.

(*) Laboratorio de Arqueobotánica. Departamento de Prehistoria. C.E.H. CSIC. Duque de Medinaceli, 8. 28014 Madrid.

El artículo fue remitido en su versión final el 26-V-97.

\section{INTRODUCCIÓN}

La Antracología (del griego Anthracos: carbón) es una disciplina naturalista cuya base de estudio es el carbón resultante de una combustión incompleta de maderas de árboles y arbustos.

El fuego que origina estos restos puede obedecer a causas naturales (incendios) o antrópicas. En el primer caso, los carbones se encontrarán en depósitos naturales (suelos geológicos), más o menos fosilizados dependiendo de sus condiciones de conservación y de su antigüedad. El análisis de carbones procedentes de paleosuelos, se ha denominado Pedo-Antracología (Thinon, 1992) y quedaría englobado íntegramente dentro de las Ciencias de la Tierra.

En el segundo caso, los restos antracológicos se encuentran en yacimientos arqueológicos como resultado de fuegos de intencionalidad diversa que los grupos humanos efectuaron durante la ocupación de los hábitats a lo largo de toda la Prehistoria. El estudio de carbones procedentes de contextos arqueológicos quedaría, de este modo, a caballo entre las Ciencias de la Tierra y las Humanidades, dando origen, junto a la $\mathrm{Pa}$ linología y la Carpología, a la denominada Arqueobotánica.

Las relaciones de las sociedades prehistóricas con su entorno natural más inmediato están en el centro de la problemática del análisis antracológico (Uzquiano, 1992a, b, 1994), a través de la 
búsqueda constante de combustible para alimentar sus fuegos cotidianos. Estas relaciones se traducen en una serie de trayectos hacia formaciones vegetales diversas para las recogidas de leña. La dirección de estos trayectos dependerá tanto de la abundancia, disponibilidad e inmediatez de estos recursos, como del sistema económico practicado por cada sociedad en todo momento. La selección de la madera estará siempre condicionada por estos factores (Uzquiano, 1992a).

En el presente trabajo se presenta la metodología de estudio de esta disciplina aplicada a la Arqueología. Los datos obtenidos dentro de este ámbito han permitido conocer la historia de la vegetación, del clima y de las relaciones hombremedio.

\section{LA MADERA OBJETO DE ESTUDIO}

La madera que el hombre trae al yacimiento puede agruparse en dos categorías funcionales: combustible y de manufactura para la construcción, la talla de objetos artesanales, etc. La primera facilita información de carácter paleoecológico; la segunda, de carácter paleoetnológico o etnobotánico (Vernet, 1992; Marguerie, 1992). De todas las categorías, la madera combustible es la que posee una mejor connotación ecológica de cara a la reconstrucción de los paleo-paisajes (Vernet, 1992a) y es este el punto de partida de todos los trabajos de síntesis antracológica realizados por la Escuela de Montpellier (Vernet, 1992b).

A través de la identificación botánica de especies leñosas (árboles y arbustos) y teniendo en cuenta su ecología, se obtiene la reconstrucción del paisaje vegetal que caracterizaba el entorno de los hábitats humanos. De este modo podrá trazarse la evolución y variaciones que las formaciones han ido sufriendo a lo largo de los períodos de la Prehistoria, como consecuencia tanto de las fluctuaciones climáticas (Paleolítico y Epipaleolítico) como de la presión del hombre sobre el medio desde el Neolítico en adelante (Thiébault and Vernet, 1987; Heinz, 1990, 1991; Vernet, 1991).

Todas las maderas en general pueden ser utilizadas como leña, aunque según sus características (porosidad, densidad, etc.), su calidad como combustible es variable. Esta simple constatación nos está indicando que tambien la leña es objeto de selección por parte del hombre (Uzquiano, 1992a, b). Las diversas categorías de la madera nos ofrecen una información diferente que no hay que prejuzgar. Un buen conocimiento de sus propiedades y cualidades favorecerá las interpretaciones antracológicas.

Los estudios etnológicos así como aquéllos procedentes de la economía prehistórica son necesarios para un mejor desarrollo de la Antracología. Sin embargo, no siempre encontramos con facilidad la información adecuada para cada análisis antracológico debido a que aquélla se halla dispersa, no publicada, o no goza de una buena difusión.

El contexto arqueológico nos proporciona una información inmediata que nos ayudará a comprender la significación de la mayor o menor variabilidad de los espectros antracológicos (Uzquiano, 1992a).

El hombre es el principal protagonista de las actividades que suponen la realización de fuegos diversos y de una serie de recogidas de leña en torno a los yacimientos habitados. Los fuegos, las recogidas de leña y los trayectos recorridos para tal fin introducen un «filtro cultural» que condicionará siempre el espectro antracológico resultante a la vez que ponen de manifiesto la relación directa que el hombre ha mantenido con el medio. Esto siempre ha sido así sea cual sea el período prehistórico/protohistórico y la región de estudio considerados (Thiébault, 1987, 1988; Uzquiano, 1992a; Otto, 1993).

\section{MÉTODOS}

La metodología de estudio se divide en dos fases. La primera tiene lugar en el yacimiento, donde los carbones son recogidos en el curso de las campañas de excavación. La segunda, se desarrolla en el laboratorio y consiste en la determinación anatómica de las muestras antracológicas y en el tratamiento de datos e interpretación de los mismos.

\section{En el terreno}

La recogida de carbones tiene que ser lo más completa posible con el fin de obtener buenos resultados tanto a nivel cuantitativo como cualitativo. Existen varias técnicas de recuperación de to- 

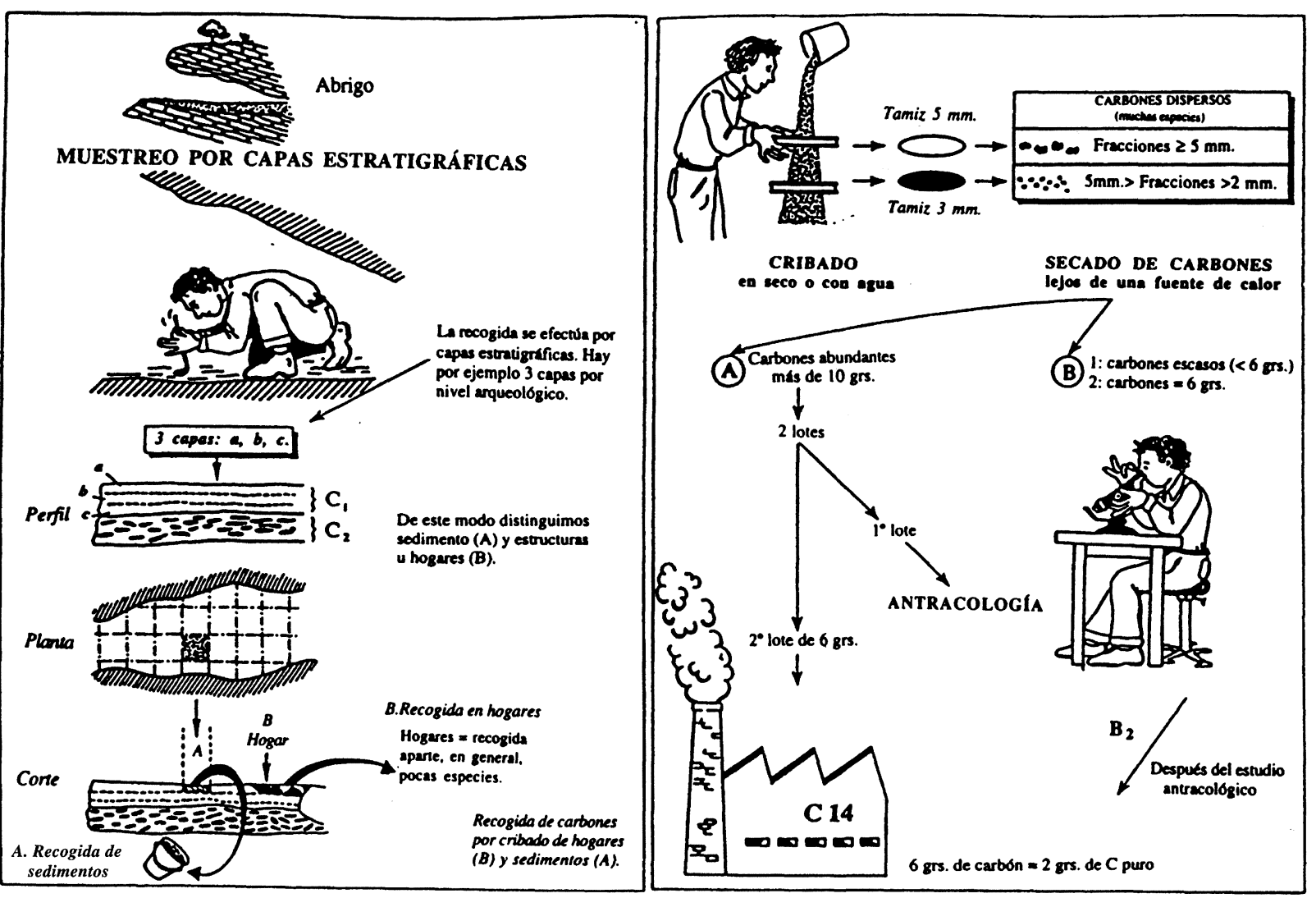

Fig. 1. Procedimientos metodológicos de la Antracología (Vernet, 1991).

dos los restos orgánicos que pueden ser encontrados en un yacimiento.

La conservación de carbones en el sedimento varía de una zona a otra. Los yacimientos situados en la región mediterránea occidental han ofrecido siempre bastantes restos ya que los carbones suelen ser fácilmente reconocibles a simple vista si bien éstos se recogen siguiendo un muestreo sistemático del nivel arqueológico mediante el cribado del sedimento en seco (Chabal, 1982). Sin embargo, las zonas húmedas de la Región Eurosiberiana, sometidas a regímenes de tipo oceánico, no ofrecen posibilidades tan óptimas para la conservación de los restos (Uzquiano, 1992a). Estos no suelen ser fácilmente visibles por ser más pequeños y, en el caso de las cuevas cantábricas, se encuentran impregnados de arcilla resultando muy delicada su extracción.

Estos factores limitan la abundancia de muestras antracológicas, por lo que la recuperación debe seguir un protocolo mucho más exhaustivo.

\section{A. Técnicas de recogida}

Los carbones visibles se recogen con la mano y van depositándose en una bolsa o similar debidamente etiquetada. Pero la gran mayoría de los carbones suele ser de pequeño tamaño y buena parte de éllos pasaría desapercibida si no se emplearan técnicas de cribado y de flotación de manera sistemática para todo el sedimento (Fig. 1). Nuestra experiencia en el terreno aconseja que desde el inicio de la excavación se integren estas dos modalidades de recuperación ya que, de lo contrario, un buen número de otros restos orgánicos pasarían desapercibidos (semillas, microfauna, insectos, etc.).

La recuperación de carbones por flotación manual es bien simple. El sedimento se introduce en una cubeta llena de agua donde se vierte calgón para disolver los carbonatos. Los carbones flotan y van separándose del sedimento con suavidad y sin fragmentarse. Se recuperan con ayuda de un colador y se depositan en un recipiente 
debidamente etiquetado. El secado de las muestras se efectúa bien al aire libre o mediante un secador de aire caliente procurando que la fuente de calor no sea demasiado fuerte. En los casos donde el sedimento es particularmente denso y los carbones muy pequeños, la flotación no es suficiente ya que un buen número de éllos no sube a la superficie. Éstos se recuperan mediante la siguiente etapa de cribado con agua. El sedimento se dispone en cribas de $5 \mathrm{~mm}$ para la fracción gruesa y en cribas de $2 \mathrm{~mm}$ para la fracción fina, lavándose cuidadosamente con agua corriente, evitando la fuerte presión de la misma. Las cribas se dejan secar y los carbones van recogiéndose durante la labor de triado o selección de todos los restos orgánicos e inorgánicos. Todos estos pasos: recogida a mano, flotación, cribado y tría de sedimentos, deben ser empleados en yacimientos en cueva al tratarse de muestras muy frágiles para ser extraídas de su sedimento directamente. En asentamientos al aire libre, generalmente de ambiente más seco, la recogida a mano y el cribado en seco o con agua son suficientes. Es aconsejable lavar el sedimento ya que el grado de fragilidad de los carbones disminuye una vez que éstos han sido mojados y secados.

El tipo de asentamiento, su funcionalidad, y contexto cultural y económico influirán de manera diferencial en la intensidad de ocupación y frecuentación del sitio (Uzquiano, 1992a). Todo ello repercutirá en la aparición de los carbones en la superficie excavada, así como en el espectro antracológico resultante (Uzquiano, 1992a, 1994). La presencia del antracólogo en el yacimiento es muy necesaria sobre todo en las primeras campañas de excavación. Tendrá primero que familiarizarse con el entorno natural del yacimiento: topografía, estudio detallado de la repartición de las formaciones vegetales, toma de muestras de maderas actuales para la colección de referencia y conocimiento del contexto arqueológico. Tambien pondrá en marcha las tareas de muestreo sistemático implicando en ellas a todos los miembros de la excavación.

\section{B. Concentración-dispersión}

A medida que la operación de muestreo y recogida va realizándose, es necesario tener en cuenta la distribución espacial de los carbones en la superficie de excavación del yacimiento (Fig. 1). Estos suelen aparecer concentrados en estruc- turas de combustión culinarias o de tipo más especializado como los hornos cerámicos o metalúrgicos y estructuras funerarias; o bien diseminados por todo el nivel de ocupación excavado, producto de anteriores concentraciones en estructuras. Estos últimos reflejan un muestreo más completo de la vegetación pasada (riqueza específica), ya que son el producto tanto de diversas recogidas de leña efectuadas de manera cotidiana en torno al hábitat, como de los diversos vaciados de hogares realizados en un lapso de tiempo no determinado («durée d'occupation», Chabal, 1991). Los concentrados en hogares, por el contrario, al representar los últimos restos de combustión, suelen ser pobres en especies y la información ofrecida no suele ser tan completa como la de los carbones dispersos. Por tanto, la distinción entre concentrados y dispersos es tarea obligada durante la fase de recogida de carbones. Este presupuesto teórico es la base de numerosos trabajos antracológicos recientes (Chabal, 1982, 1988, 1991; Heinz, 1990, 1991; Badal, 1992; Figueiral, 1990), y ha sido tenido en cuenta por nosotros solamente durante la fase de recogida atendiendo a la distribución espacial, procedencia de carbones y contexto arqueológico. L. Chabal si bien tiene en cuenta las recogidas de leña en torno al hábitat y la duración de la ocupación del mismo, en el momento de interpretar los resultados, se olvida de los condicionantes de toda índole que influenciaron aquéllas recogidas. Esta línea creada por ella (Chabal, 1982) y continuada en los otros trabajos (Badal, 1992; Heinz, 1990, 1991) ha generado numerosas críticas en el seno de la Etnoarqueología. Estos estudios se basan en un tratamiento estadístico exxhaustivo de unos datos que ofrecen una información muy limitada sobre las relaciones hombre-medio por estar sacados de su contexto.

Para nosotros, sin embargo, la idea de selección por parte del hombre está siempre presente tanto en carbones dispersos como concentrados. La mayor o menor diversidad en especies, y por tanto la selección de maderas reflejada en cada espectro antracológico, dependerá siempre de factores tanto naturales (topografía) como socioeconómicos (variedad del entorno en recursos disponibles y buscados, funcionalidad del yacimiento, tipo de estacionalidad). Sólo el estudio en profundidad del contexto arqueológico así como del marco geográfico donde se ubica cada yacimiento, podrán proporcionarnos aquella in-

T. P., 54, n. ${ }^{\circ} 1,1997$ 
formación. En este sentido, es más importante la realización de un estudio del modo de repartición de las muestras en la superficie excavada, que la simple distinción entre concentración y dispersión de carbones. Pero este trabajo deberá ir orientado a la reconstrucción y organización del hábitat en torno al fuego, y no únicamente a saber si los carbones presentan o no una distribución homogénea en el área excavada o bien cuál es la superficie mínima de muestreo (Heinz, 1990; Badal, 1992).

\section{Antracología y C-14}

La madera carbonizada ha sido generalmente recogida para efectuar dataciones por radiocarbono, siendo éstas necesarias. Al igual que ocurre en la palinología de lagos y turberas (Van Campo, 1976), las interpretaciones y atribuciones de los espectros resultarían peligrosas si no se contara con fechas, dadas las oscilaciones climáticas que han sucedido durante el Pleniglaciar. Ahora bien, situar cronológicamente un espectro no implica su atribución climática sin más. Esta última vendrá dada por la discusión previa acerca de la significación ecológica de las especies aparecidas.

En yacimientos donde la abundancia de carbones sea considerable se aconseja hacer dos lotes: uno iría directamente al laboratorio de radiocarbono, y el otro, al laboratorio de análisis paleobotánicos. Sin embargo, en yacimientos donde la escasez de estos restos sea manifiesta, se aconseja el análisis antracológico previo a la datación ya que la muestra, al no ser destruida, puede ser recuperada para ser datada posteriormente (Fig. 1). En contra de lo que muchos arqueólogos piensan el carbón, al no pasar por ningún tratamiento químico, no se contamina (Vernet et alii, 1979).

Lo ideal sería efectuar la determinación botánica previa a la datación, seleccionando por un lado, los taxa supuestamente acordes con cada período concreto; por otro, aquéllos considerados «problemáticos» (por ej. los mesotermófilos que aparecen en períodos fríos). Con ello se pretende confirmar cronológicamente la contemporaneidad de todos los taxa con su nivel correspondiente. En caso de producirse distorsiones de fechas, podrían detectarse las zonas de excavación con percolaciones de material antracológico. Esta operación debería ser obligada en los yacimien- tos del Paleolítico superior, especialmente si se sabe con seguridad que éstos han sufrido alteraciones en sus procesos postdeposicionales.

\section{En el laboratorio: análisis antracológico}

Los carbones, una vez recogidos, son trasladados al laboratorio donde comienza la segunda etapa metodológica.

\section{A. Determinación botánica}

El análisis se basa en la determinación botánica de la madera carbonizada en función de sus características anatómicas. El carbón se secciona con la mano orientando cada fractura hacia los tres planos anatómicos o secciones transversal, longitudinal tangencial y longitudinal radial (Fig. 2). Cada una de estas tres secciones es observada en un microscopio de reflexión (OLYMPUS BH o BXM). Esta técnica sencilla y rápida permite estudiar un gran número de fragmentos sin precisar ningún tipo de manipulación química previa.

Las técnicas de preparación de las muestras en láminas delgadas ya pertenecen a la historia de la Antracología (Couvert, 1970; Santa, 1961; Santa et Vernet, 1968). Estos procedimientos, si bien persisten aún en algún laboratorio, fueron abandonados a partir de los años 60 (Stieber, 1967) y 70 (Vernet, 1973; Castelletti, 1975) por ser muy lentos en el estudio de un gran número de mues-

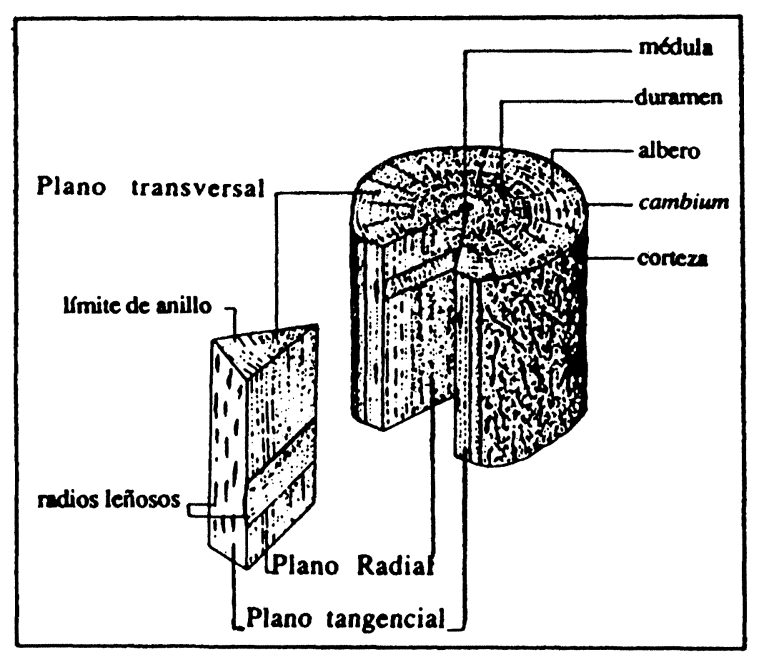

Fig. 2. Secciones y planos anatómicos de la madera (Marguerie, 1992). 
tras e imprecisos en las determinaciones botánicas, dado el gran número de manipulaciones químicas empleadas. Las muestras determinadas pueden ser recuperadas para efectuar dataciones radiométricas (Vernet et alii, 1979), o bien observadas en el Microscopio Electrónico de Barrido (M.E.B.) si se necesita una mayor precisión en la identificación de ciertos detalles anatómicos, dudosos en el microscopio óptico.

La determinación se efectúa siguiendo una serie de claves de identificación establecidas en diversos Atlas de anatomía de maderas no carbonizadas (Greguss, 1955, 1959; Jacquiot, 1955; Jacquiot et alii, 1973; Schweingruber, 1978, 1990). Es necesario contar con una colección de referencia de especies actuales carbonizadas por no existir en la actualidad atlas de anatomía de carbones publicados.

La determinación genérica y específica se consigue de manera más o menos rápida para un gran número de taxa. Sin embargo, existen algunas limitaciones en lo referente a las claves de identificación que contribuyen a que esta fase de trabajo sea delicada y laboriosa. Las Leguminosas presentan un problema a nivel de género por falta de caracteres precisos de diagnosis para diferenciar una especie de otra. En estos casos se recomienda utilizar las maderas de leguminosas actuales recolectadas en el entorno de cada yacimiento y carbonizarlas para poder identificar mediante comparaciones. En nuestro caso (yacimientos paleolíticos de la zona cantábrica, Uzquiano, 1992a), hemos podido determinar los géneros Cytisus y Ulex y, dentro de éste último, Ulex europaeus y Ulex gallii .

Ciertas Ericáceas (Erica sp.) del cortejo atlántico tambien pueden presentar problemas de determinación específica, sobre todo si el ma-terial no se ha conservado en buenas condiciones.

En el caso del género Juniperus, sí existen claves para la distinción específica en los atlas de anatomía (Greguss, 1955). A pesar de ello, en todos los trabajos antracológicos conocidos, Juniperus aparece generalmente sin especificación.

La identificación específica del género Salix es mucho más delicada debido a la gran variedad de especies actuales que cohabitan dentro de su formación característica. Ciertos atlas de anatomía así lo sugieren (Schweingruber, 1978).

En el caso de Quercus, la determinación específica es ambigua cuando se hace referencia a dos especies (por ej. Quercus robur-petraea) por las hibridaciones de que son objeto los diversos robles caducifolios, observables en la gran variedad de sus planos leñosos.

\section{B. Tratamiento de datos}

En esta etapa los datos se trabajan cualitativa y cuantitativamente, aplicando diversas pruebas estadísticas.

\section{Unidad de medida}

La unidad de base considerada es el fragmento de carbón sea cual sea su tamaño (Cha-bal, 1982, 1990). Otros estudios han conside-rado sólo como unidad la masa media (Thinon, 1979; Castelletti, 1975) o ambas unidades de cara a comparar los resultados obtenidos (Kraus-Marguet, 1981; Chabal, 1982; Bazile-Robert, 1982).

' La fragmentación de cada carbón es siempre aleatoria e inexplicable mostrando una gran variabilidad de un taxon a otro (Chabal, 1982). Cada uno presenta una sobrefragmentación o una subfragmentación que influye considerablemente en las frecuencias relativas del número de fragmentos (Chabal, 1988, 1991). Los recientes estudios llevados a cabo en la reducción de masa y pérdida de peso (Loreau, 1994) intentan aproximarse al problema de la fragmentación todavía no resuelto mediante la combustión experimental.

No obstante, los estudios comparativos fragmento-masa media convergen en resultados paleoecológicos equivalentes. La identificación botánica es más cómoda de obtener fragmento a fragmento que por cada gramo de carbón (Chabal, 1982), por lo que la hemos adoptado.

\section{Número de fragmentos}

La interpretación antracológica se basa en la variación de las frecuencias relativas de cada taxon determinado, siendo necesario saber el número mínimo de carbones que tendrán que estudiarse para que la imagen de la vegetación sea fiable. Las curvas taxonómicas que, en principio, sirven para ilustrar el mero análisis cualitativo, se elaboran para poner en relación la riqueza específica obtenida con el número de fragmentos analizados. A lo largo de la curva se observa siempre que el número de taxa aumenta a medida que el número de fragmentos analizados es mayor. La curva tiende a estabilizarse a partir de cierto um-

T. P., 54, n. ${ }^{\circ} 1,1997$ 
bral que oscila ente 100,250 y 600 fragmentos (Bazile-Robert, 1982; Chabal, 1982, 1988, 1991; Heinz, 1990). Este presupuesto metodológico ha sido tenido en cuenta, reiteradamente en cada trabajo de síntesis antracoanalítica efectuado (Figueiral, 1990, etc.). Las curvas taxonómicas inéditas que hemos realizado presentaban una gran variabilidad. En un trabajo reciente (Uzquiano, 1994) utilizamos dos ejemplos de estas curvas un tanto extremos para minimizar la rigidez metodológica de este presupuesto. A nuestro parecer el número de fragmentos no es un principio metodológico en sí. La mera presencia/ausencia de los taxa, no es una información despreciable, teniendo en cuenta no sólo el problema de la fragmentación, sino que tambien el tipo de depósito en muchos casos, no ha permitido conservar un gran número de carbones.

Una curva taxonómica nos pone en evidencia taxa que pertenecen a formaciones vegetales de ecología diversa, dependientes de las características del medio natural y de la selección intencionada con que se haya operado sobre las mismas. En lugares donde la topografía sea un factor determinante, y el terrritorio sea recorrido y explotado por el hombre de manera múltiple, puede obtenerse una gran variedad de taxa con sólo muy pocos fragmentos (Fig. 3a); pero donde los condicionantes sean climáticos (Fig. 3b), analizar un número elevado de muestras no es muy útil.

\section{Interpretación de los resultados}

Los resultados florísticos obtenidos, cuyas frecuencias relativas están representadas gráficamente en un diagrama, tienen que ser interpretados en términos de vegetación. Para ello es necesario tener un conocimiento profundo de la vegetación potencial en torno al yacimiento y de la repartición de las asociaciones vegetales a nivel de toda la región de estudio. El punto de partida es la vegetación actual y de ahí se pasa a considerar el comportamiento de los taxa de manera individual (autoecología) y en relación con su asociación vegetal (sinecología). La Interpretación Ecológica Preliminar de carbones dispersos y concentrados es siempre posible. Esta pone de manifiesto la paleovegetación y las condiciones ambientales que existían en el entorno de los yacimientos.

La utilización de determinadas especies de una o varias formaciones nos sugiere además la selección que se opera en el interior de éstas por
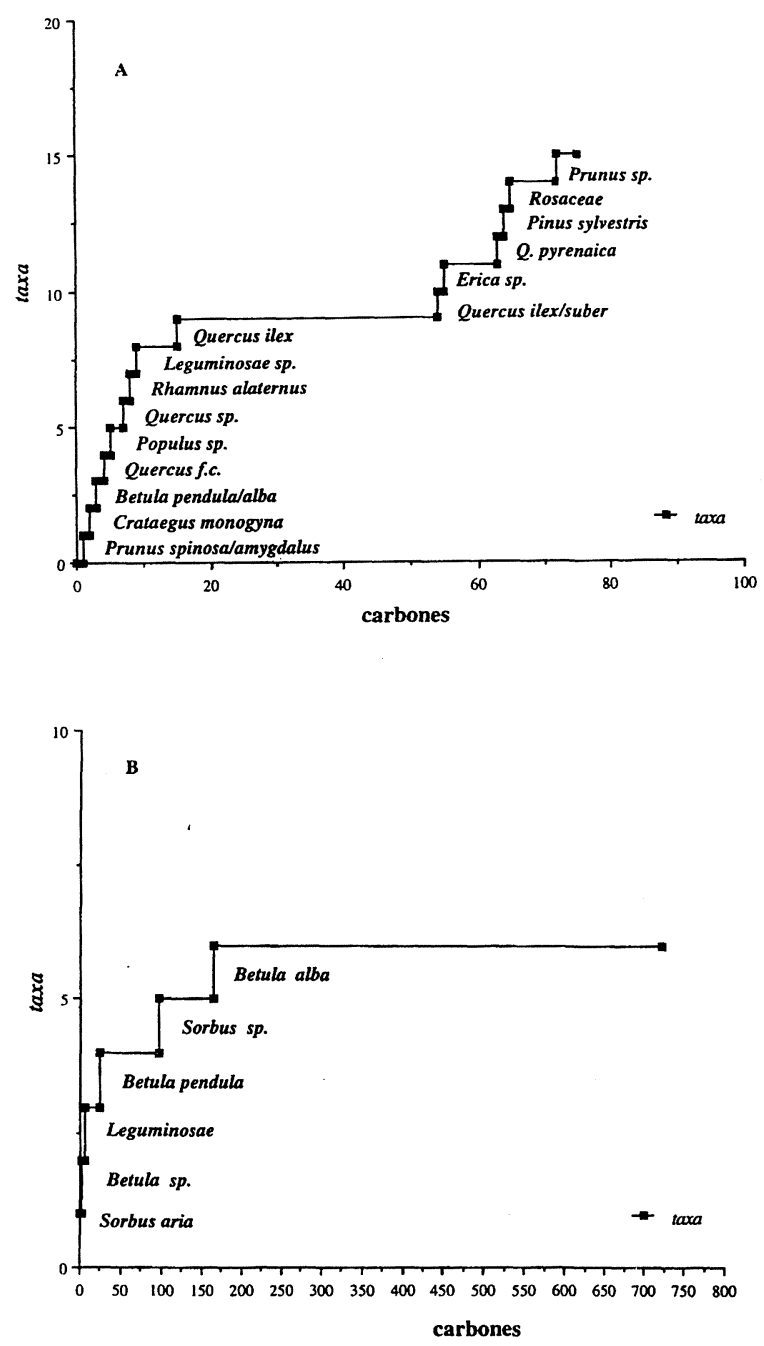

Fig. 3. Curvas taxonómicas que ilustran la riqueza específica de cada análisis antracológico: 3a. Curva obtenida en el lecho 21 de la cueva de Santa Catalina, Bizkaia. 3b. Curva del subnivel 18c de la Cueva de El Castillo, Cantabria (Uzquiano, 1994).

parte del hombre obedeciendo tanto a causas físicas (clima, medio físico) como socioeconómicas (duración de ocupación del hábitat, funcionalidad, etc.). Un estudio en profundidad de estas causas conduce a integrar en la interpretación toda la información procedente del contexto arqueológico. De este modo, entramos en el capítulo Relaciones Hombre-Medio sobre la base de la Economía Prehistórica (Uzquiano, 1992a, b, 1995a). Los paralelos etnográficos de sociedades actuales pueden aportarnos tambien buena infor- 
mación, si bien su búsqueda resulta bastante difícil y los datos existentes son muy escasos en lo referente a búsqueda de leña.

Lo que en definitiva es importante es tener en cuenta las necesidades económicas que en cada momento de la Prehistoria han configurado el sistema de relaciones del hombre con su entorno natural. Su desarrollo supondría admitir claramente la selección de la madera, no sólo en casos concretos (Thiébault, 1988; Heinz, 1993), sino en general, con un estudio de las distancias recorridas para su recolección (Uzquiano, 1992a, b, 1995a; Otto, 1993). Sin embargo este capítulo es un mero enunciado en la Escuela de Montpellier en la cual la consideración de la selección queda únicamente reservada a los carbones encontrados en el interior de estructuras de combustión, presuponiendo que la pobreza específica es sinónimo de una fuerte intervención humana, cuando la selección de madera está ya implícita en su recolección. Ello conduce a interpretaciones arbitrarias, contradictorias y, en algunos casos, muy discutibles. El estudio de las relaciones hombremedio debe preceder a la Discusión Paleoecológica, de cara a evaluar mejor las aportaciones de la Antracología en el terreno de la Paleoecología.

Dentro del enfoque paleoecológico debe tenerse en cuenta el comportamiento histórico de cada taxon: la modalidad de aparición, la extensión y el papel desempeñado en cada ciclo climático del pasado, no ha sido siempre igual (Watts, 1988). Aquí entramos de lleno en la vertiente $\mathrm{Pa}$ leobotánica. La recurrencia constante a referencias bibliográficas sobre este particular es una tarea obligada que implica una labor de búsqueda permanente, llevando al antracólogo al contacto con otras disciplinas naturalistas (por ej. las secuencias polínicas de lagos y turberas o el registro de los fondos oceánicos).

La necesidad constante de combustible por parte del hombre ligada al tipo de movilidad efectuado («logistical mobility»/«logistical expeditions», Straus, 1987, 1991) ha puesto de manifiesto una serie de taxa particulares (Uzquiano, 1992a, 1995b), que han logrado mantenerse debido a condiciones de abrigo: zonas refugio a partir de las cuales estas especies pudieron extenderse una vez que fuesen favorecidas climáticamente (Webb, 1986; Huntley \& Webb, 1989). De la misma manera se han evidenciado taxa ya extintos (caso de Picea en la zona Cantábrica, Uzquiano, 1995a).

T. P., 54, n. ${ }^{\circ} 1,1997$

\section{CONCLUSIONES}

Hemos definido la Antracología como disciplina paleobotánica y arqueobotánica en virtud de sus numerosas implicaciones en áreas diversas de las Ciencias, ya sean Experimentales o Históricas. En su vertiente arqueobotánica, las maderas objeto de estudio sea cual sea su categoría tienen para nosotros una doble connotación, paleoecológica y paleoetnológica, indisociable. Los grupos humanos están implicados en su recogida, bien diaria como leña para fuegos de funcionalidad diversa, o bien buscada con alguna finalidad particular por adecuarse mejor a la manufactura de objetos varios, a la construcción e incluso el combustible. En cualquiera de estos casos, la madera siempre es objeto de selección y está condicionada por una serie de factores. Las características del medio físico configurarán de entrada la abundancia o escasez de los recursos, su accesibilidad y su proximidad o lejanía y, por consiguiente, la naturaleza de los trayectos recorridos en su búsqueda. Los factores climáticos así como los socioeconómicos completarán toda esta serie de condicionantes, llegando incluso a determinar la elección del asentamiento mismo (Fig. 4). Pero el hombre siempre es y está en el

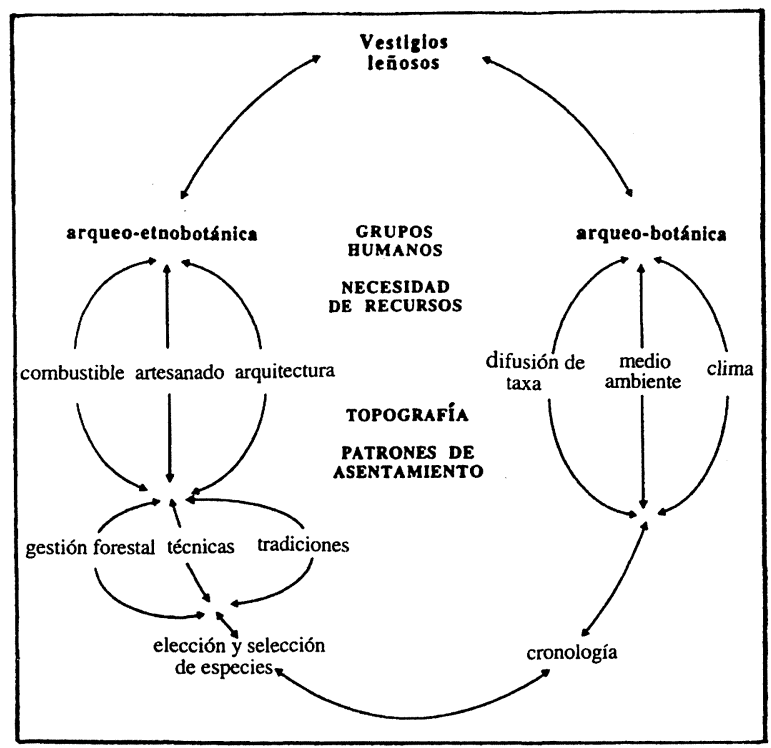

Fig. 4. Organigrama que integra las diferentes lecturas o niveles de interpretación del análisis antracológico referidas en el texto (Marguerie, 1992) con los principales factores determinantes propuestos por nosotros. 
centro de la problemática antracológica que es la que a nosotros nos importa (Uzquiano, 1992a).

La madera combustible es la que efectivamente ofrece mejores posibilidades en el terreno de la Paleoecología. Pero tomar esta vía no implica dejar de lado la connotación paleoetnológica que la leña lleva implícita tanto en carbones dispersos como en los concentrados. Si las recogidas de leña (en caso de haberse estudiado) obedecen a una misma naturaleza de trayectos, la diversidad o pobreza específica resultante se verá reflejada en los resultados obtenidos. La observación que L. Chabal (1991: 21) apunta a nivel teórico sobre los hogares culinarios y la duración de ocupación es muy acertada, si bien le reprochamos el que no incluya la selección humana ni el contexto arqueológico de donde provienen los carbones. Sus principios teórico-metodológicos caen en una rigidez formal en el momento de sus aplicaciones. Teoría y práctica parecen discurrir por caminos diferentes.

La percepción, utilización y modificación del entorno natural, así como el modo de moverse dentro de éste han variado a lo largo del tiempo en función de los recursos buscados por los grupos humanos en cada momento: en una economía de subsistencia, en el paso hacia una economía de producción, etc.

Un análisis antracológico es objeto de diferentes lecturas. La mayor o menor riqueza interpretativa dependerá de los aspectos que queramos poner de relieve. Pero para nosotros todos ellos forman un conjunto indisociable, producto de una realidad compleja que tendemos a simplificar por el hecho de haber tenido lugar en un pasado más o menos lejano.

\section{BIBLIOGRAFÍA}

BAdAL García, E. (1992): «L'anthracologie préhistorique: à propos de certains problèmes méthodologiques». En J.L. Vernet (éd.): Les Charbons de bois, les anciens écosystèmes et le rôle de l'homme. Bulletin de la Societé Botanique de France, 139. Actualités Bottaniques, 2/3/4: 167-189.

Badal, E.; Bernabé, J. \& Vernet, J.L. (1994): «Vegetation changes and human action from the Neolithic to the Bronze Age (7000-4000 BP) in Alicante, Spain, based on charcoal analysis». Vegetation History \& Archaeobotany, 3: 155-166.

BAzILE-RoberT, E. (1982): «Données experimentales pour l'anthracoanalyse». Etudes Quaternaires Languedociennes, 2: 19-28.

Castelletti, L. (1975): «Depositi mesolitico del passo della Comunelle (reggio E.), Apennino Tosco-Emiliano». Preistoria Alpina, 11: 148-154.

Chabal, L. (1982): Méthodes de prélèvement des bois carbonisés protohistoriques pour l'étude des relations homme-végétation (exemple d'un habitat de l'âge du fer: Le Marduel, St. Bonnet du Gard, fin VIIIèmefin Ier s. av. J.C. Mémoire de D.E.A. Ecologie végétale. U.S.T.L. Montpellier II.

- (1988): «Pourquoi et comment préléver les charbons de bois pour la période antique: les méthodes utilisées sur le site de Lattes (Hérault)». Lattara, 1: 187-222.

- (1991): L'homme et l'évolution de la végétation méditerranéenne, des âges de métaux à la période romaine: Recherches anthracologiques théoriques, appliquées principalement à des sites du Bas Languedoc. Thèse Physiologie et Biologie des Organismes et des Populations. U.S.T.L. Montpellier.

COUVERT, M.,(1970): Etude des charbons de bois préhistoriques. Preparation des coupes minces et analyse des structures. Travaux du Centre de Recherches Anthropologiques, Préhistoriques, Ethnographiques. Alger A.M.G. Paris.

Figueiral, I. (1990): Le Nord-Ouest de Portugal et les modifications de l'Ecosystème, du Bronze final à l'époque romaine, d'après l'anthracoanalyse de sites archéologiques. Thèse Physiologie et Biologie des Organismes et des Populations. U.S.T.L. Montpellier.

GreGUSS, P. (1955): Identification of living Gymnospermes on the basis of xylotomy. Akadémiai Kiadó. Budapest.

- (1959): Holzanatomie der Europaïschen Laubhölzer und Sträucher. Akadémiai Kiadó.Budapest.

Heinz, C. (1990): Dynamique des végétations holocènes en Méditerranée Nord-Occidentale d'après l'anthracoanalyse de sites préhistoriques: Méthodologie et Paléoécologie. Paléobiologie Continentale, XVI (2). U.S.T.L. Montpellier II.

- (1991): «Upper Pleistocene and Holocene vegetation in the south of France and Andorra. Adaptations and first ruptures: new charcoal analysis data». Review of Palaeobotany \& Palynology, 69 (1): 299-324.

- (1993): «La vegetación de los Pirineos orientales en relación con las actividades humanas desde el Neolítico medio hasta el Bronce final». Estudios sobre Cuaternario: 199-204.

Huntley, B. \& WebB, T. III (1989): «Migration species' reponse to climatic variations caused by changes in the Earth's orbit». Journal of Biogeography, 16: 519.

JACQUIOT, C. (1955): Atlas d'anatomie des bois des conifères. Centre Technique du Bois CNRS. Paris.

JACQuiot, C.; TREnARD, Y. et Dirol, D. (1973): «Atlas 
d'anatomie des bois des Angiospermes (essences feuillues)». Centre Technique du Bois, CNRS. Paris.

LOREAU, P. (1994): Du bois au charbon de bois : approche experimentale de la combustion. Mémoire de D.E.A. Environnements et Archéologie. U.S.T.L. Montpellier II.

MARguerie, D. (1992): «Le bois: Evolution, Structure et détermination». En D. Marguerie et J.Y. Hunot (eds.): Les bois archéologiques. Association Grand Ouest pour la Recherche en Archéo-Sciences, A.G.O.R.A., 2: 3-8. Université de Rennes I.

Отто, T. (1993): Phyto-archéologie des sites archéologiques de l'âge du Fer au Diamare, nord du Cameroun: le site de Salak, étude de bois et grains carbonisés. Thèse Biologie de Populations et Ecologie, Environnements et Archéologie. U.S.T.L., 2 vols. Montpellier II.

Perles, C. (1977): Préhistoire du feu. Masson \& Cia. Paris.

SANTA, S. (1961): «Essai de reconstitution de paysages végétaux quaternaires de l'Afrique du Nord». Lybica, 6-7: 37-77.

Santa, S. et Vernet, J.L. (1968): «Une technique de préparation des charbons de bois préhistoriques en vue de leur étude anatomique». Naturalia Montpeliensia, 19: 171-177.

SCHWEINGRUBER, F. (1978): Mikroskopische holzanatomie. Zücher A. G. Zug. Switzerland.

- (1990): Anatomie europäischer Hölzer. WSL/FNP Haupt. Switzerland.

Straus, L.G. (1987): «Hunting in late-upper Palaeolithic Western Europe». En H. Nitecki \& D.V. Nitecki (eds.): Evolution of Human hunting. Plenum. London: 147-176.

- (1991): «Epipalaeolithic and Mesolithic adaptations in cantabrian Spain and pyrenean France». Journal of World Prehistory, 5 (1): 83-104.

STIEBER, J. (1967): «A Magyaroszàgi Felsöpeisztocén vegetátió törnete az anthrakotómiai eredmények Tükreben». Födtani Közlony, 97 (3): 308-317.

Thiebault, S. (1987): «L'analyse des charbons de bois: une méthode d'approche des relations de l'homme avec son milieu végétal». Archéologie en Aquitaine. Bulletin de liaison et d'information, 6: 126-131.

- (1988): «L'homme et le milieu végétal. Analyse anthracologiques de six gisements de Pré-Alpes au Tardiglaciaire et au Postglaciaire». Documents d'Archéologie Française, 15. Maison des Sciences de l'Homme. Paris.

Thinon, M. (1979): Incidence écologique des reboisements du Mont Ventoux (Vaucluse). Aspects floristiques et pédologiques. Thèse 3ème cycle. Ed. Université d'Aix-Marseille III.

- (1992): L'Analyse Pédoanthracologique. Aspects Méthodologiques et Applications. Thèse Sciences Biologiques. Ed. Université d'Aix-Marseille III.
UzQUiAno, P. (1992a): Recherches anthracologiques dans le secteur Pyréneo-cantabrique (Pays Basque, Cantabria et Asturias): Environnements et relations homme-milieu au Pléistocène supérieur et début de l'Holocène. Thèse Biologie des Organismes et Populations, Environnements et Archéologie. U.S.T.L. Montpellier II.

- (1992b): «L'homme et le bois au Paléolithique en Région Cantabrique, Espagne. Les exemples d'Altamira et El Buxu». En J.L. Vernet (éd.): Les charbons de bois, les anciens écosystèmes et le rôle de l'homme. Bulletin de la Société Botanique de France, 139. Actualités Botaniques, 2/3/4: 361-372.

- (1994): «Ciencias del Cuaternario. La Antracología». Investigación y Ciencia, 210: 32-34.

- (1995a): «La disparition de Picea à la fin du Pléistocène supérieur en région cantabrique d'après l'anthracoanalyse: déterminisme climatique et anthropique». Comptes Rendus Acadadémie des Sciences, 321, série IIa. Paris: 545-551.

- (1995b): «L'évolution de la végétation à l'Holocène initial dans le Nord de l'Espagne à partir de l'étude anthracologique de trois sites archéologiques». Quaternaire, 6 (2): 77-83.

VAN CAMPO, M. (1976): «La méthode pollenanalytique en archéologie». En H. de Lumley (éd.): La Préhistoire Française C.N.R.S. Paris: 463-464.

VERNET, J.L. (1990): «The bearing of phyto-archaeological evidence on discussions of climatic change over recent millennia». Philosophical Transactions of the Royal Society of London A, 330: 671-677.

- (1991): «L'histoire du paysage humanisé révèlée par les bois carbonisés». En J. Guilaine (éd.): Pour une archéologie agraire. Armand Colin. Paris: 369-408.

- (1992): «Charbons de bois et Paléoenvironnent Méditerranéen». En D. Marguerie et J.Y. Hunot (éds.): Les bois archéologiques, A.G.O.R.A., 2. Université de Rennes I: 21-25.

- (éd.):Les Charbons de Bois, les anciens ecosystèmes et le rôle de l'homme. Colloque Montpellier 10-13 Septembre 1991. Bulletin de la Société Botanique de France, 139.Actualités Botaniques, 2/3/4.

Vernet, J.L. \& Thiebault, S. (1987): «An approach to northwestern mediterranean recent prehistoric vegetation and ecological implications». Journal of Biogeography, 14: 117-127.

Vernet, J.L.; Bazile-Robert, E. et Evin, J. (1979): «Coordination des analyses anthracologiques et des datations absolues sur charbons de bois». Bulletin de la Société Préhistorique Française, 76: 76-79.

WATTs, W.A. (1988): «Late Tertiary and Pleistocene vegetation history: Europe». En B. Huntley \& T. Webb III (eds.):Vegetation History. Kluwer,Dordrecht: 155-192.

WeBB, T. III (1986): «Is vegetation in equilibrium with climate? How to interpret late-Quaternary pollen data». Vegetatio, 67: 75-91. Dordrecht.

T. P., 54, n. ${ }^{\circ} 1,1997$ 